\title{
Acute myocardial infarction associated with abacavir and tenofovir based antiretroviral drug combinations in the United States
}

\author{
Kunchok Dorjee ${ }^{1,2^{*}}$, Manisha Desai ${ }^{3,4}$, Tsering Choden $^{5}$, Sanjiv M. Baxi ${ }^{6}$, Alan E. Hubbard ${ }^{1,7}$ and \\ Arthur L. Reingold ${ }^{1}$
}

\begin{abstract}
Introduction: Although individual antiretroviral drugs have been shown to be associated with elevated cardiovascular disease (CVD) risk, data are limited on the role of antiretroviral drug combinations. Therefore, we sought to investigate CVD risk associated with antiretroviral drug combinations.

Methods: Using an administrative health-plan dataset, risk of acute myocardial infarction (AMI) associated with current exposure to antiretroviral drug combinations was assessed among persons living with HIV receiving antiretroviral therapy (ART) across the U.S. from October 2009 through December 2014. To account for confounding-by-indication and for factors simultaneously acting as causal mediators and confounders, we applied inverse probability of treatment weighted marginal structural models to longitudinal data of patients.

Results: Over 114,417 person-years ( $n=73,071$ persons) of ART exposure, 602 cases of AMl occurred at an event rate of 5.26 (95\% Cl: 4.86, 5.70)/1000 person-years. Of the 14 antiretroviral drug combinations studied, persons taking abacavir-lamivudine-darunavir had the highest incidence rate (IR: 11/1000; 95\% Cl: 7.4-16.0) of AMI. Risk (HR; 95\% Cl) of AMI was elevated for current exposure to abacavir-lamivudine-darunavir (1.91; 1.27-2.88), abacavir-lamivudineatazanavir $(1.58 ; 1.08-2.31)$, and tenofovir-emtricitabine-raltegravir $(1.35 ; 1.07-1.71)$. Tenofovir-emtricitabine-efavirenz was associated with reduced risk $(0.65 ; 0.54-0.78)$. Abacavir-lamivudine-darunavir was associated with increased risk of AMI beyond that expected of abacavir alone, likely attributable to darunavir co-administration. We did not find an elevated risk of AMI when abacavir-lamivudine was combined with efavirenz or raltegravir.

Conclusion: The antiretroviral drug combinations abacavir-lamivudine-darunavir, abacavir-lamivudine-atazanavir and tenofovir-emtricitabine-raltegravir were found to be associated with elevated risk of AMI, while tenofoviremtricitabine-efavirenz was associated with a lower risk. The AMI risk associated with abacavir-lamivudine-darunavir was greater than what was previously described for abacavir, which could suggest an added risk from darunavir. The results should be confirmed in additional studies.
\end{abstract}

Keywords: Human Immunodeficiency Virus, Antiretroviral agents, Cardiovascular disease

*Correspondence: kdorjee1@jhmi.edu

${ }^{1}$ Division of Epidemiology, School of Public Health, University of California Berkeley, Berkeley, CA, USA

Full list of author information is available at the end of the article

\section{Introduction}

Over the last decade, there has been an extraordinary increase in the number of people living with HIV (PLHIV) receiving antiretroviral therapy (ART), with that number increasing from 8 million in 2010 to 25.4 million by 2019 [1]. Combination ART has been pivotal in original author(s) and the source, provide a link to the Creative Commons licence, and indicate if changes were made. The images or other third party material in this article are included in the article's Creative Commons licence, unless indicated otherwise in a credit line to the material. If material is not included in the article's Creative Commons licence and your intended use is not permitted by statutory regulation or exceeds the permitted use, you will need to obtain permission directly from the copyright holder. To view a copy of this licence, visit http://creativecommons.org/licenses/by/4.0/. The Creative Commons Public Domain Dedication waiver (http://creativeco mmons.org/publicdomain/zero/1.0/) applies to the data made available in this article, unless otherwise stated in a credit line to the data. 
reducing HIV/AIDS-related mortality ratios from 5.9\% (1.7 million HIV/AIDS related deaths out of 28.7 million PLHIV) in 2004 to $1.8 \%$ (690,000 HIV/AIDS related deaths out of 38 million PLHIV) in 2019 [1]. As ART options expand and costs of treatment decline, understanding the toxicities associated with the use of antiretroviral agents is needed to improve health outcomes of PLHIV.

There is ongoing debate on the association of several contemporary antiretroviral agents with increased risk of cardiovascular disease, including abacavir [2-8], lamivudine [8, 9], atazanavir [9], and darunavir [10, 11]. While most antiretroviral agents are thought to contribute to cardiovascular disease by worsening blood lipid levels, other biological mechanisms, such as platelet reactivity, insulin resistance and lipodystrophy, have been reported as the underlying mechanisms for cardiotoxicity [12]. Additionally, a channeling bias may affect the observed associations between antiretroviral exposure and cardiovascular outcomes [5, 9, 13-15]. Antiretroviral medications are universally prescribed in combinations, but to date few studies have systematically evaluated the risk of cardiovascular disease associated with exposure to antiretroviral drug combinations; most CVD risk assessments have been made for individual drugs [9].

In this study, we investigated the relationship between receipts of various antiretroviral drug combinations containing agents from four major drug classes, including nucleos(t)ide reverse transcriptase inhibitors (NRTI), non-nucleoside reverse transcriptase inhibitors (NNRTI), protease inhibitors (PI), and integrase strand transfer inhibitors (InSTI), used in the treatment of HIV infection and the subsequent risk of acute myocardial infarction (AMI).

\section{Methods}

\section{Study population and data}

Risk of AMI associated with exposure to combinations of antiretroviral drugs was assessed among PLHIV $\geq 18$ years of age started on ART between October 1, 2009 and December 31, 2014 and enrolled in the IMS' PharMetrics Plus database in the United States. QuintilesIMS PharMetrics Plus is one of the largest health plan insurance claims databases in the U.S., containing adjudicated claims for more than 100 million unique enrollees from four regions of the U.S. [16]. In the U.S., during the study period, $98 \%$ of PLHIV were insured and thus represented by this claims dataset [16, 17]. An algorithm specified a priori was used to extract and define the study population (Fig. 1). Individuals who had not received any ART were excluded from the study. October 1, 2009 was the earliest possible date for complete availability of relevant data; ART prescription history was not available prior to this date. The baseline time point was defined as the date of ART initiation in the database and individual follow-up time was censored at the first of three events after baseline: (1) first occurrence of AMI, (2) last recorded date of ART receipt, 3) December 31, 2014. The study was approved by the Committee for Protection of Human Subjects at the University of California, Berkeley.

\section{Exposure and outcome}

Individual antiretroviral drugs in the database were identified by their unique generic product identifier (gpi) codes. Any two prescriptions for a given antiretroviral agent separated by $<30$ days were combined to represent a single continuous exposure; gaps $\geq 30$ days were treated as separate ART exposures and not considered as a single continuous exposure. Start and stop dates are available for each antiretroviral drug; this enables determination of whether a specific antiretroviral drug(s) was switched to another drug. Exposure time for a drug combination was generated based on whether an individual was receiving the component drugs simultaneously, determined by examining the start and stop dates of the individual drugs. Of 23 drug combinations defined a priori, 14 combinations were assessed based on availability of adequate exposure-time ( 1500 person-years), ascertained by examining the duration of exposure given by the dates of initiation and stopping of the antiretroviral agent in the database. Each drug combination consisted of three (or four if boosted) drugs: two NRTIs and either a boosted PI, an NNRTI, an InSTI or another NRTI. A separate analytical dataset was generated for each exposure. The data were longitudinal and each subject's follow-up time was divided into consecutive one-month periods during which treatment status was allowed to vary. Current exposure to a drug combination was defined as exposure (yes/no) during each one-month observation period. The exposure time of a specific drug combination was compared to exposure-time of all other antiretroviral agents in assessing the outcome. The outcome was defined as the first occurrence of acute myocardial infarction after baseline, represented in the database by International Classification of Disease, 9th Revision, Clinical Modification (ICD-9-CM) code 410. xx. (Additional file 1: Table S1).

\section{Statistical analysis}

The risk of AMI associated with current exposure to a given drug combination was assessed using stabilized inverse probability of treatment weighted (sIPTW) marginal structural models [18]. To construct sIPTWs, four pooled logistic regression models were used, two each for the denominator and numerator as previously described 


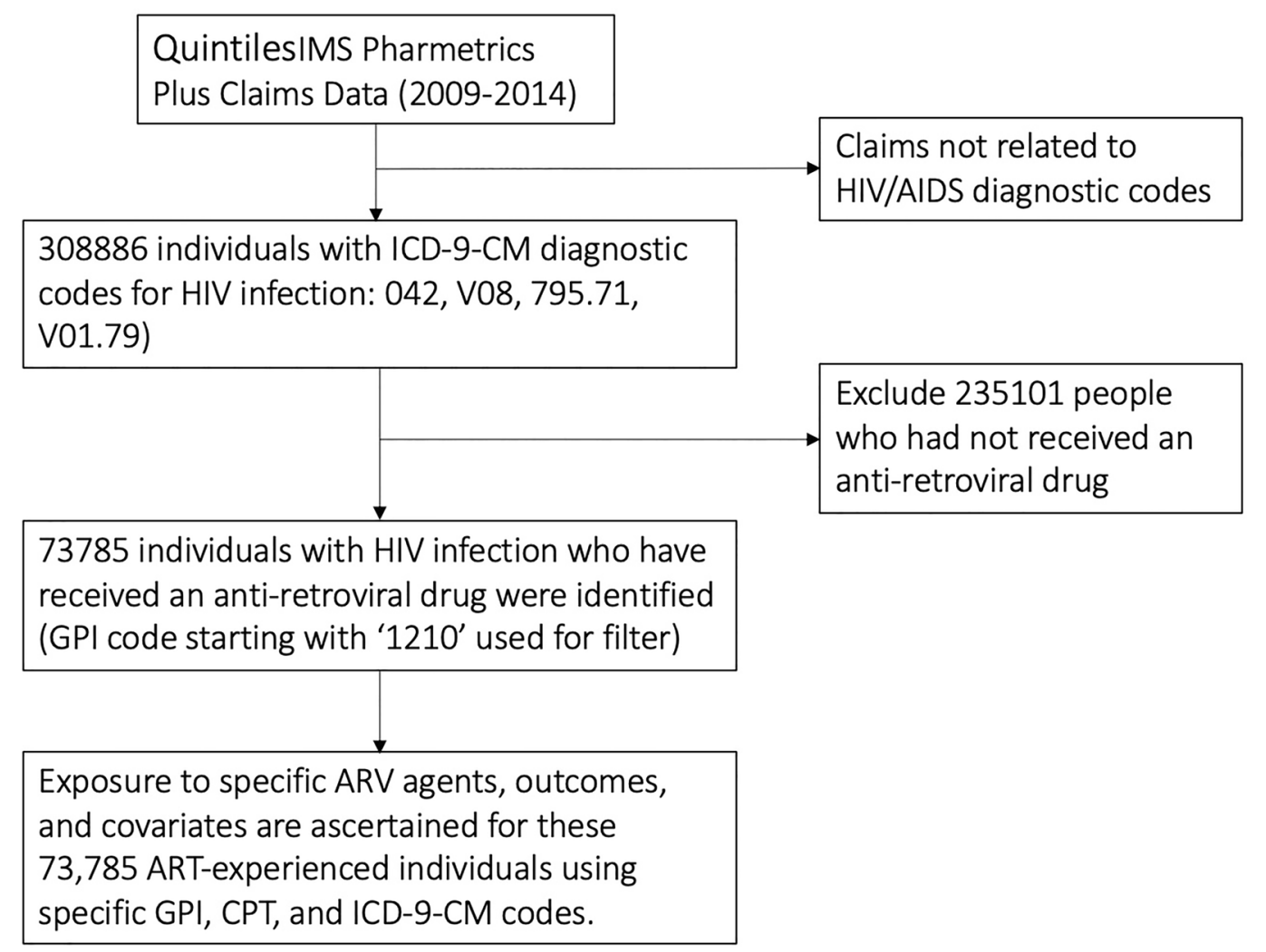

GPI: PLWHIV: people living with HIV; Generic Product Identifier; CPT: Current Procedural Terminology; ICD-9-CM: International Classification of Disease, $9^{\text {th }}$ Revision, Clinical Modification.

Fig. 1 Algorithm for identification of study subjects in the Quintiles IMS Pharmetrics Plus claims data for study of cardiovascular disease associated with antiretroviral therapy

$[5,15]$. For the denominator, the probability of exposure initiation was first modelled as a function of baseline and time-dependent covariates. Pooled logistic regression model was fit to data up to the individual's first month of receiving the exposure or the end of follow-up for those never exposed. The probability of continuing exposure was then modelled by fitting the model to data after the first month of exposure initiation. The treatment continuation model differed from the treatment initiation model in additionally containing a variable for past month's exposure status. The probabilities for the numerator of the SIPTW were modelled as a function of baseline covariates only. The baseline or time-fixed covariates were sex, tobacco use (ever), substance use (ever), alcohol abuse (ever), chronic hepatitis $\mathrm{B}$ and $\mathrm{C}$ virus infections, history of stroke, history of cancer, and prior myocardial infarction. The time-dependent covariates were age, treatment year, body mass index (BMI), receipt of antihyperglycemic agents (sulfonylureas, biguanides, insulin, thiazolidinedione), receipt of cardiovascular medications (aspirin, beta-blocker, angiotensin converting enzyme inhibitor, angiotensin receptor blocker, calcium channel blocker, and statins), and diagnosis of chronic kidney disease (CKD), dyslipidemia, heart failure, cardiac dysrhythmia, atherosclerosis, diabetes mellitus, or hypertension. Cumulative exposure to each of abacavir, lamivudine, tenofovir, emtricitabine, zidovudine, atazanavir, and darunavir was additionally incorporated in the treatment models to generate weights excluding drugs contained in the drug-combination under investigation. Followup time was modelled as a function of natural cubic splines with three internal knots at 25th, 50th, and 75th percentiles.

The final marginal structural hazard model was adjusted for the sIPTW and baseline values of the following covariates: baseline age, sex, year of treatment start in the database, smoking, use of alcohol, and substance, prior heart disease or receipt of medications for heart disease, lipodystrophy, hypertension, chronic kidney disease, diabetes mellitus, and dyslipidemia. Standard errors for the treatment and marginal models were calculated robustly to account for repeated measures within 
an individual. We performed sensitivity analyses to determine variables to include into the treatment model and assess if the models yield qualitatively different results, as described by Cole and Hernan [19].

In secondary analyses, conventional Cox proportional hazard models were run for each exposure, adjusting for baseline and time-dependent variables that included: age, treatment year, tobacco use (ever), substance use (ever) and the following conditions at baseline: stroke, hepatitis $\mathrm{B}$ or $\mathrm{C}$ virus infections, any cancer, prior heart disease or receipt of cardiovascular medications, lipodystrophy, categorical BMI status, chronic kidney disease, diabetes mellitus, and dyslipidemia. We did not adjust for multiple comparisons, as we had a priori hypotheses based on findings in the published literature and a separate dataset was meticulously generated to test each exposure; hence, such adjustments would be unnecessarily conservative. The data were extracted from the claims databases using TERADATA (Dayton, $\mathrm{OH}$ ), and SAS version 9.1 (SAS Institute, Cary, NC). The marginal structural models were implemented in STATA version 13.1 (StataCorp, College Station, TX) following methods described by Fewell et al. [20]. We have provided additional details of the marginal structural Cox proportional hazard models in a prior publication [5].

\section{Results}

\section{Population and exposure}

The database contained 114,417 person-years of exposure-time to ART contributed by 73,071 study participants. AMI occurred in 602 individuals at an event rate of 5.26 (95\% CI: 4.86, 5.70) per 1000 person-years. The median age of the study population was 45 years and $81.5 \%$ were male. The median follow-up time ranged from 2.67 years for individuals receiving tenofoviremtricitabine-darunavir to 3.58 years for those tenofovir-lamivudine-zidovudine/efavirenz. The demographic and clinical characteristics of the participants overall and stratified by AMI outcome are shown in Table 1. Traditional cardiovascular disease risk factors, i.e., higher age, male sex, hypertension, diabetes mellitus, dyslipidemia, smoking, substance abuse, pre-existing heart disease, and chronic kidney disease were more prevalent among individuals who developed AMI. Fourteen antiretroviral drug combinations, each representing $\geq 1500$ personyears of exposure-time, were studied (Table 2). Incidence rates [IR $(95 \% \mathrm{CI})$ ] of AMI ranged from 3.4/1000 (3/1000-4/1000) people for tenofovir-emtricitabineefavirenz to $11.0 / 1000(7.4 / 1000-16 / 1000)$ people for abacavir-lamivudine-darunavir.

\section{Risk of AMI}

Using sIPTW marginal structural models, we found significantly elevated risk of AMI for current exposure to abacavir-lamivudine-darunavir (adjusted hazard ratio: 1.91; 95\% CI: $1.27-2.88, \mathrm{p}=0.002$ ), abacavir-lamivudineatazanavir (aHR: 1.58; 95\% CI: 1.08- 2.31, $\mathrm{p}=0.019$ ), and tenofovir-emtricitabine-raltegravir (1.35; 1.07-1.71, $\mathrm{p}=0.010$ ). Forest plot and Kaplan Meier Survival curves for the risk of acute myocardial infarction associated with the antiretroviral drug combinations are shown in Figs. 2 and 3. Risk was not significantly elevated for the remaining regimens, including: abacavir-lamivudine-efavirenz, abacavir-lamivudine-raltegravir, abacavir-lamivudinezidovudine, tenofovir-lamivudine-zidovudine, tenofovirlamivudine-efavirenz, tenofovir-lamivudine-efavirenz, tenofovir-lamivudine-darunavir, tenofovir-lamivudineatazanavir, and tenofovir-emtricitabine-fosamprenavir. Risk of AMI was lower for people receiving tenofoviremtricitabine-efavirenz (HR: 0.65; 95\% CI: 0.54-0.78, $\mathrm{p}<0.001)$. Similar results were obtained in secondary analyses using conventional Cox models (Additional file 1: Table S2).

\section{Discussion}

Using a large nationally representative real-world health plan dataset containing longitudinal information of more than 70,000 PLHIV receiving care across the United States, we have described the results of AMI risk for various antiretroviral drug combinations, several of which were recommended initial regimens. We found an elevated risk of AMI associated with current exposure to three antiretroviral drug combinations: abacavir-lamivudine-darunavir, abacavir-lamivudine-atazanavir, and tenofovir-emtricitabine-raltegravir. The incidence rate and hazard ratio were highest for abacavir-lamivudinedarunavir (IR: 11/1000 person-years; HR: 1.91) and lowest for tenofovir-emtricitabine-efavirenz (IR: 3.40/1000 person-years; HR: 0.65). The population level estimate for the annual incidence of myocardial infarction in the U.S. is $1.86 / 1000$ people [21], whereas the AMI incidence rate for individuals receiving abacavir-lamivudine-darunavir approached the incidence rate for people $\geq 75$ years of age in the U.S. (8.53-15.90/1000 person-years) [21]. In a prospective multi-national cohort (The Data Collection on Adverse Events of Anti-HIV Drugs [D:A:D]), the investigators calculated an incidence rate of $13.67 / 1000$ person-years for PLHIV who received darunavir [11]. The association between Abacavir use and cardiovascular disease was first reported by the D:A:D study group in 2008 [8]. 
Table 1 Baseline characteristics of adults with HIV infection, with and without acute myocardial infarction (AMI) receiving antiretroviral therapy in the Pharmetrics Plus data from October 2009 through December 2014 in the United States

\begin{tabular}{|c|c|c|c|}
\hline Characteristics & $\begin{array}{l}\text { All patients }(\mathrm{N}=73,071), \mathrm{n} \\
(\%)\end{array}$ & $\begin{array}{l}\text { Patients without AMI }(\mathrm{N}=72,469) \text {, } \\
\mathrm{n}(\%)\end{array}$ & $\begin{array}{l}\text { Patients with AMI } \\
(\mathrm{N}=602), \mathrm{n}(\%)\end{array}$ \\
\hline Age, median (IQR) & $45(38-52)$ & $45(38-52)$ & $53(48-59)$ \\
\hline Male gender & $59,514(81.4)$ & $58,981(81.4)$ & $533(88.5)$ \\
\hline \multicolumn{4}{|l|}{ Region } \\
\hline East & $17,523(24.0)$ & $17,354(24.0)$ & $169(28.1)$ \\
\hline Mid-West & $13,532(18.5)$ & $13,411(18.5)$ & $121(20.1)$ \\
\hline South & $33,271(45.5)$ & $33,014(45.6)$ & $257(42.7)$ \\
\hline West & $8745(12.0)$ & $8690(12.0)$ & $55(9.1)$ \\
\hline \multicolumn{4}{|l|}{ Year of ART initiation } \\
\hline 2009 & $24,435(33.4)$ & $24,127(33.3)$ & $308(51.2)$ \\
\hline 2010 & $9729(13.3)$ & $9636(13.3)$ & $93(15.5)$ \\
\hline 2011 & $10,274(14.1)$ & $10,190(14.1)$ & $84(14.0)$ \\
\hline 2012 & $8605(11.8)$ & $8559(11.8)$ & $46(7.6)$ \\
\hline 2013 & $7830(10.7)$ & 7794 (10.8) & $36(6.0)$ \\
\hline 2014 & $12,198(16.7)$ & $12,163(16.8)$ & $35(5.8)$ \\
\hline Ever substance abuse & $13,395(18.3)$ & $13,152(18.2)$ & $243(40.4)$ \\
\hline Ever alcohol abuse & $3093(4.2)$ & $3039(4.2)$ & $54(9.0)$ \\
\hline Ever tobacco use & $11,849(16.2)$ & $11,587(16.0)$ & $262(43.5)$ \\
\hline Body mass index $>24.9$ & $1511(2.07)$ & $1503(2.07)$ & $8(1.33)$ \\
\hline Essential hypertension & $7450(10.2)$ & $7336(10.1)$ & $114(19.0)$ \\
\hline $\begin{array}{l}\text { Diabetes mellitus or receipt of anti-hyperglycemic } \\
\text { agents }^{\mathrm{a}}\end{array}$ & $4128(5.7)$ & $4052(5.6)$ & $76(12.6)$ \\
\hline Chronic Kidney Disease & $729(1.0)$ & $701(1.0)$ & $28(4.7)$ \\
\hline Dyslipidemia & $8616(11.8)$ & 8496 (11.7) & $120(19.9)$ \\
\hline Lipodystrophy & $224(0.3)$ & $220(0.3)$ & $4(0.7)$ \\
\hline Pre-existing heart disease $\mathrm{e}^{\mathrm{b}}$ & $1754(2.4)$ & $1688(2.3)$ & $66(11.0)$ \\
\hline Receipt of medications for heart disease ${ }^{c}$ & $14,336(19.6)$ & $14,074(19.4)$ & $262(43.5)$ \\
\hline History of stroke & $211(0.3)$ & $204(0.3)$ & $7(1.2)$ \\
\hline Hepatitis B & $959(1.3)$ & $953(1.3)$ & $6(1.0)$ \\
\hline Hepatitis C & $1520(2.1)$ & $1500(2.1)$ & $20(3.3)$ \\
\hline History of cancer & $5499(7.5)$ & $5442(7.5)$ & $57(9.5)$ \\
\hline
\end{tabular}

a Sulfonylureas, biguanides, insulin, thiazolidinedione

${ }^{b}$ Prior myocardial infarction, heart failure, cardiac arrhythmia, and atherosclerosis

${ }^{c}$ Aspirin, beta-blockers, calcium channel blockers, statins, angiotensin converting enzyme inhibitors, angiotensin receptor blockers

\section{Abacavir-lamivudine based combinations}

There is an accumulating body of evidence suggesting an elevated risk of CVD associated with use of abacavir $[3-8,15]$. PLHIV taking abacavir have previously been shown to have a $43 \%$ increase risk, using this same database [5]. The finding of a 58\% increase in risk of AMI associated with the antiretroviral drug combination abacavir-lamivudine-atazanavir is in keeping with the $61 \%$ increased risk described for abacavir exposure in a recent systematic review and meta-analysis [6]. Our analysis suggests a higher risk when abacavir and lamivudine were combined with the protease inhibitor darunavir (HR: 1.91), which may suggest an incremental additive effect of darunavir. Although an analysis of data from Janssen-sponsored clinical trials suggested no elevation in risk of AMI with darunavir use [10], a CVD risk elevation of $60 \%$ for exposure to darunavir was observed in the D:A:D cohort [11]. Our finding of elevated risk for abacavir-lamivudine-atazanavir agreed with that of Desai et al. in a U.S. Veterans Affairs (VA) population [9]. The finding of no increased risk for abacavir-lamivudine-efavirenz was also observed in a prior study using a separate commercial health plan data-set in the United States that reported a significantly lower CVD risk for those taking an efavirenz-containing antiretroviral regimen, an elevated risk for efavirenz free regimens, and no risk when 
Table 2 Median years of exposure and number of acute myocardial infarction (AMI) events occurring over total person-time of exposure to combinations of anti-retroviral agents

\begin{tabular}{|c|c|c|c|c|}
\hline Antiretroviral drug & $\begin{array}{l}\text { Median no. of years of exposure } \\
\text { (IQR) }\end{array}$ & No. of AMI events & $\begin{array}{l}\text { Person years } \\
\text { of exposure }\end{array}$ & $\begin{array}{l}\text { Incidence rate per } \\
1000 \text { people }(95 \% \\
\mathrm{Cl})\end{array}$ \\
\hline ABC-3TC-ATV & $2.75(1.58,4.17)$ & 30 & 3951 & $7.6(5.3-8.7)$ \\
\hline ABC-3TC-DRV & $2.83(1.58,4.08)$ & 26 & 2395 & $11.0(7.4-16)$ \\
\hline ABC-3TC-ZDV & $2.92(1.75,4.33)$ & 19 & 2882 & $6.6(4.2-10.3)$ \\
\hline ABC-3TC-EFV & $2.92(1.75,4.25)$ & 9 & 2541 & $3.5(1.8-6.8)$ \\
\hline ABC-3TC-RAL & $3.08(1.75,4.12)$ & 20 & 3055 & $6.5(4.2-10.1)$ \\
\hline TDF-3TC-ZDV & $3.58(2.25,4.67)$ & 32 & 5549 & $5.8(4.1-8.2)$ \\
\hline TDF-3TC-EFV & $3.58(2.33,4.58)$ & 18 & 4529 & $4.1(2.5-6.3)$ \\
\hline TDF-3TC-DRV & $3.16(2.00,4.33)$ & 19 & 2336 & $8.1(5.2-12.8)$ \\
\hline TDF-3TC-ATV & $3.25(1.92,4.42)$ & 23 & 2843 & $8.1(5.4-12.2)$ \\
\hline TDF-FTC-ATV & $2 \cdot 83(1.67,4.17)$ & 63 & 13,620 & $4.6(3.6-5.9)$ \\
\hline TDF-FTC-DRV & $2 \cdot 67(1.50,4.83)$ & 62 & 10,981 & $5.6(4.4-7.2)$ \\
\hline TDF-FTC-EFV & $2.92(1.75,4.33)$ & 170 & 49,493 & $3.4(3.0-4.0)$ \\
\hline TDF-FTC-FPV & $3 \cdot 17(1.83,4.42)$ & 13 & 2465 & $5.3(3.1-9.1)$ \\
\hline TDF-FTC-RAL & $2 \cdot 83(1.67,4.17)$ & 92 & 13,729 & $6.7(5.5-8.2)$ \\
\hline
\end{tabular}

$A B C$ abacavir, TDF tenofovir dixoproxil fumarate, FTC emtricitabine, 3TC lamivudine, ATV atazanavir, DRV darunavir, FPV fosamprenavir, EFV efavirenz, RAL raltegravir

efavirenz was combined with abacavir [11, 22]. While it is unclear why abacavir-lamivudine-efavirenz was not associated with an elevated risk in this study, given that abacavir has been shown to be associated with CVD elsewhere; [6] interaction between abacavir and efavirenz may be possible although we are not aware of a biological or pharmacological rationale for such an interaction. We did not find an elevated risk of AMI for the abacavirlamivudine-raltegravir combination. While it is possible that patients with unfavorable cardiovascular risk profiles may be channeled away from receiving an abacavir based regimen including abacavir-lamivudine-raltegravir, it remains to be determined if any portion of this finding may be attributable to the lipid lowering property of InSTIs [23-25]. Our investigation of the 14 drug combinations, nine of which contained lamivudine, did not reveal a pattern indicating that lamivudine in and of itself may be associated with an elevated risk of AMI. However, the D:A:D study authors reported an increased risk of AMI associated with recent exposure to lamivudine after adjusting for past exposure, and the VA study found an elevated risk of CVD for lamivudine use, noting that all of the drug-combinations associated with an elevated CVD risk contained lamivudine $[8,9]$. The differing findings on lamivudine call for further investigations.

\section{Tenofovir-lamivudine and tenofovir-emtricitabine based combinations}

We did not find any significantly increased risk of AMI for most of the tenofovir-containing combinations, including tenofovir-lamivudine-efavirenz, tenofovir-emtricitabine-atazanavir, and tenofovir-emtricitabine-fosamprenavir, tenofovir-lamivudine-atazanavir, tenofovir-lamivudine-darunavir, and tenofovir-emtricitabine-darunavir. Our findings on tenofovir-based regimens largely agree with that of the VA study. We are not aware of a prior study that has implicated tenofovir/ emtricitabine in the development of AMI. Tenofovir has been shown to have an intrinsic lipid lowering effect and to decrease the carotid intima media thickness, suggesting possible cardiovascular and cerebrovascular protective effects [26, 27]. We observe an increased risk of AMI (HR:1.35; 95\% CI: 1.07-1.71)) when tenofoviremtricitabine was combined with raltegravir. This finding prompted us to assess the distribution of baseline CVD risk factors for the participants who did and did not receive tenofovir-emtricitabine-raltegravir. We observed that participants who received tenofovir-emtricitabineraltegravir during the follow-up period were more likely to have cardiovascular disease and dyslipidemia at baseline, compared to participants who did not receive this combination ( 14 vs $11 \%$, p value $<0.001)$. Therefore, it is possible that individuals with an adverse cardiovascular disease risk profile may have selectively been given tenofovir-emtricitabine-raltegravir, in an effort to reduce their ART-attributable CVD risk. Also, we note the possibility of advanced HIV disease, which is independently associated with CVD risk [28], in patients receiving tenofovir-emtricitabine-raltegravir. Our dataset lacks CD4 cell-count and HIV viral load data and therefore, we 


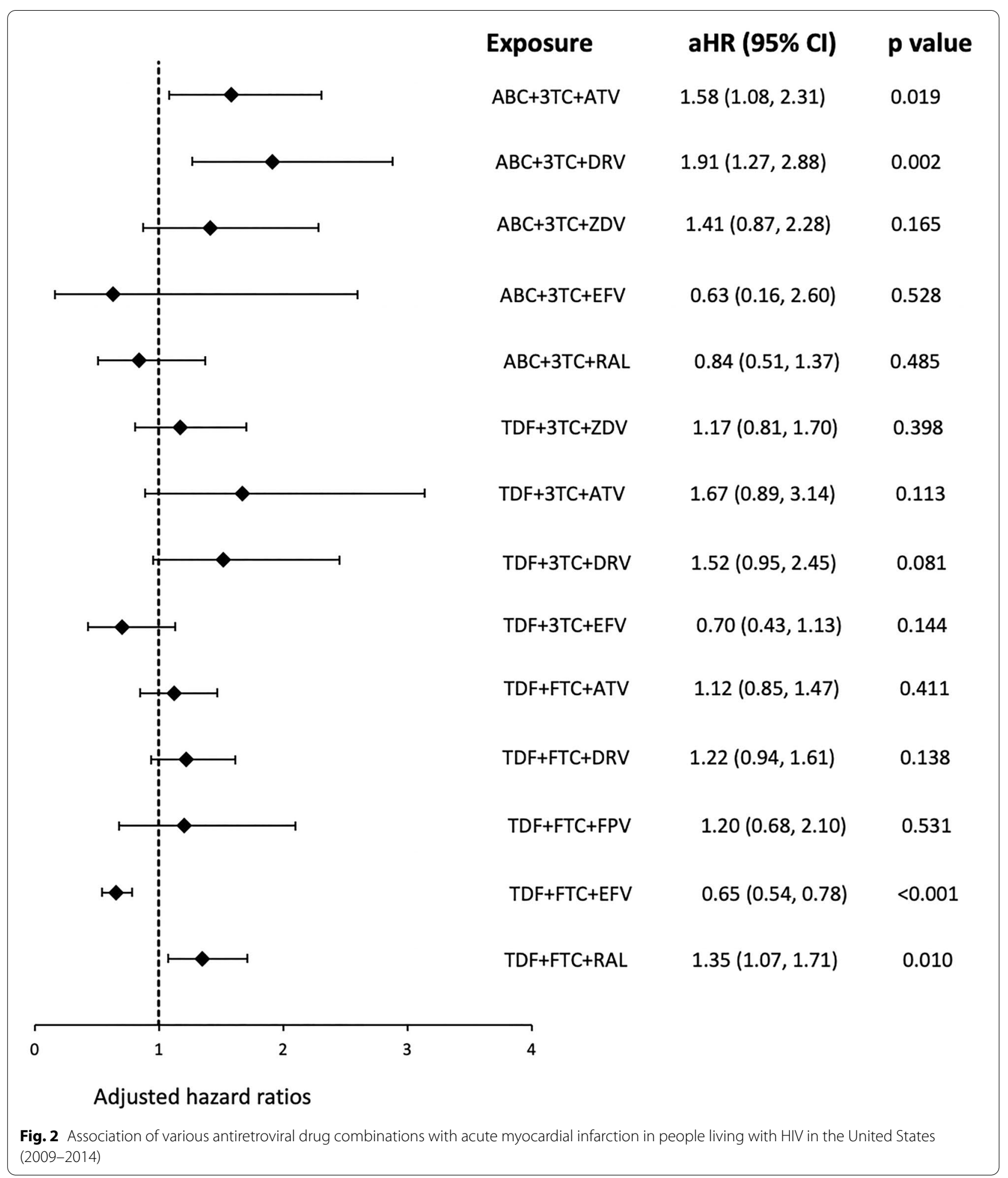

could not investigate this hypothesis. Nevertheless, we are not aware of a prior study that has investigated the risk of CVD among recipients of an antiretroviral drug that contains raltegravir, and encourage further inquiry to determine if this observation and that of a risk reduction with EFV, are due to random chance or channeling bias. 

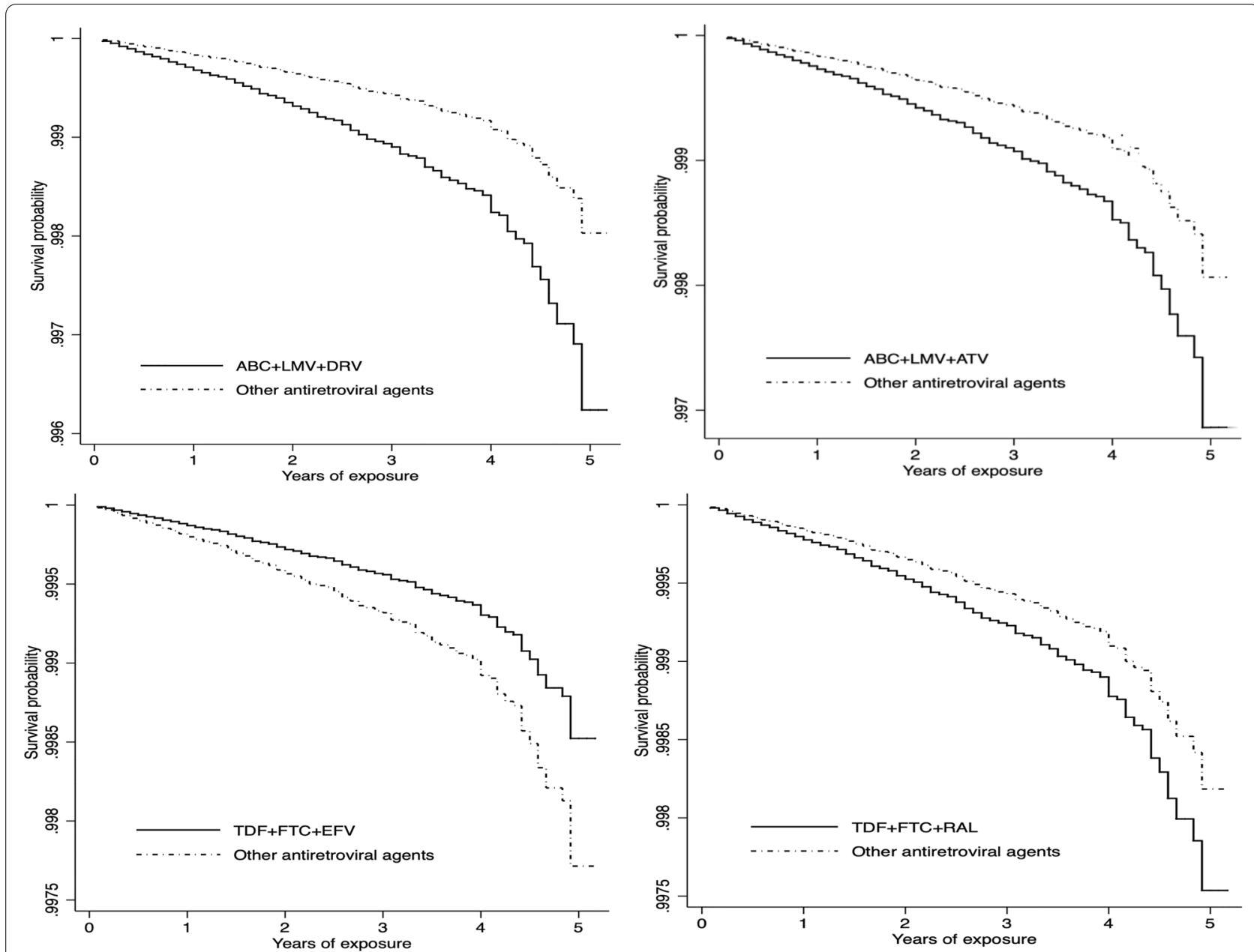

Fig. 3 Risk of acute myocardial infarction associated with use of specific antiretroviral drug combinations in the United States (2009-2014)

Our findings on the drug combinations have implications for clinical practice, as they inform the thinking about risk of CVD in the setting of combination ARTthe only way in which ART is currently used. The combination of abacavir-lamivudine and dolutegravir, an InSTI, is a recommended initial ART regimen for PLHIV. Our finding of no increased risk when abacavir-lamivudine was combined with raltegravir suggests value in further study of other InSTI-based combinations including abacavir-lamivudine-dolutegravir. However, we note that raltegravir and dolutegravir, although both being InSTIs, can be quite different in terms of their side-effect profiles [29]. We encourage further investigations and external validation of raltegravir and its combinations with abacavir-lamivudine or tenofovir-emtricitabine, especially as both combinations with InSTIs are recommended as initial ART regimens for PLHIV [30].

The claims database we used has limitations that should be noted. First, there is no information on possible risk modifiers, such as CD4 cell-count, HIV viral load and race/ethnicity. We are unsure how these factors may have influenced the prescription of a tenofovir or abacavir based antiretroviral regimen. Also, the majority of the study population are male and therefore, the results may not be readily generalizable to women living with HIV. Although, the ICD-9-CM code used to ascertain AMI outcome has been previously validated, [31] some misclassification of events may have occurred. We are unsure whether any such misclassification would have affected the direction of the associations of various antiretroviral drug combinations with CVD risk in the participants. ART prescription history prior to October 1, 2009 was also not available in the database. As a result, influence of prior exposure to the older protease inhibitors and to thymidine analogues on the subsequent development of CVD could not be assessed. Data were generated from claims submitted to the health plan for reimbursement, and it is possible that information on health conditions 
for which reimbursement may not be sought could be under-reported in the database. However, such errors are likely to affect exposure groups non-differentially, in which case any bias in the results would have been toward the null. Coding errors and missing data related to treatment adherence are most likely to have affect the exposure groups non-differentially. Finally, in the modeling, we could not have been able to adjust for unmeasured confounders or residual confounding, even with marginal structure models, which are entirely dependent on the quality of data at entry.

There are a number of strengths to this work. Most studies on CVD risk from ART exposure so far have been in clinical cohorts; validating risk in a different setting is important. Compared to prior studies, we used more recent data. Because of the possibility of confoundingby-indication where an individual receives (or not) a drug based on specific clinical indications, such as chronic kidney disease or dyslipidemia, we used inverse-probability weighted marginal structural models to account for confounding. We have reported our results from marginal structural and conventional Cox models. Although the estimates from marginal structural models and Cox models did not differ substantially, the former seeks to capture the CVD risk had all PLHIV received the given antiretroviral drug combination vs had they all not received the ART combination. Hence, the estimates from the marginal models may be more representative of the true population estimates $[18,32]$.

\section{Conclusion}

The antiretroviral drug combinations of abacavir-lamivudine-darunavir, abacavir-lamivudine-atazanavir, and tenofovir-emtricitabine-raltegravir were found to be associated with a greater risk of AMI, whereas tenofoviremtricitabine-efavirenz was found to be associated with a lower risk of AMI. The AMI risk for abacavir-lamivudine-darunavir was greater than what has been previously described for abacavir, suggesting an added risk from darunavir. The results should be confirmed in additional studies. Greater awareness of the effects of specific antiretroviral drug combinations can benefit patients with specific risk profiles, ART history, or antiretroviral drug contraindications.

\section{Supplementary Information}

The online version contains supplementary material available at https://doi. org/10.1186/s12981-021-00383-7.

Additional file 1: Table S1. ICD-9-CM, and CPT codes for defining various covariates and outcomes. Table S2. Risk of myocardial infarction associated with current exposure to various combinations of antiretroviral agents among people living with HIV in the United States
Acknowledgements

Not applicable.

\section{Disclosures}

Gilead Sciences (Foster City, CA, USA) supported the acquisition of the data. Gilead Sciences was not involved in the design and conduct of the study; collection, management, analysis, and interpretation of the data; preparation, review, and approval of the manuscript; and decision to submit the manuscript for publication.

\section{Authors' contributions}

$K D$ and $A R$ was responsible for concept and design of the study. KD analyzed the data. KD and SB interpreted the data. SB, MD, TC, AH, SB and AR reviewed the results and contributed towards analysis and interpretation of the data. $\mathrm{KD}$ and $\mathrm{SB}$ performed literature search. KD drafted the manuscript. SB, MD, TC, $A H, S B$ and AR critically reviewed the draft and provided edits. AR provided mentorship to $\mathrm{KD}$ and provided overall supervision. All authors have seen and approved the final report.

\section{Author information}

K.D. is currently Assistant Professor of Medicine at the Johns Hopkins School of Medicine Infectious Diseases. K.D. is working on global control of tuberculosis and HIV-AIDS, especially in the pediatric population. K.D. completed his Ph.D. degree in Epidemiology from UC-Berkeley School of Public Health under supervision of Arthur Reingold, MD, and Alan Hubbard, PhD, who have overseen his doctoral dissertation work including the work contained in this manuscript. Dr. Reingold, co-author on this paper, is Professor of Epidemiology and the head of UC-Berkeley School of Public Health Division of Epidemiology and Dr. Hubbard, co-author, is Professor of Biostatistics in the UC-Berkeley School of Public Health. Dr. Baxi was an infectious disease fellow at the University of California, San Francisco and a Ph.D. student at UC-Berkeley at the time of this work.

\section{Funding}

Dr. Dorjee's work is supported by US National Institute of Allergy and Infectious Diseases (Grant \# K01Al148583), Johns Hopkins Alliance for a Healthier World (Grant \# 80045453), UNOPS STOP TB PARTNERSHIP TB REACH (Grant \# 134126) and Pittsfield Anti-TB Association. Dr. Dorjee has received support from philanthropists including the Chao Family, Ms. Ann Down, and the Paster Family.

\section{Availability of data and materials} No restrictions.

\section{Declarations}

\section{Ethics approval and consent to participate}

The study was approved by the Committee for Protection of Human Subjects at the University of California, Berkeley as non-human subjects research, obviating the need for participant consent.

\section{Consent for publication}

N/A.

\section{Competing interests}

None to report for any author.

\section{Author details}

${ }^{1}$ Division of Epidemiology, School of Public Health, University of California Berkeley, Berkeley, CA, USA. ${ }^{2}$ Center for Tuberculosis Research, Johns Hopkins University School of Medicine, 1550 Orleans Street, Baltimore, MD 21287, USA. ${ }^{3}$ Quantitative Sciences Unit, Center for Biomedical Informatics Research, Department of Medicine, Stanford University, Palo Alto, CA, USA. ${ }^{4}$ Department of Biomedical Data Science, Stanford University, Palo Alto, CA, USA. ${ }^{5}$ School of Public Health, Department of Epidemiology and Biostatistics, State University of New York Downstate Medical Center, Brooklyn, NY, USA. ${ }^{6}$ Department of Epidemiology and Biostatistics, University of California San Francisco, San Francisco, CA, USA. 'Division of Biostatistics, School of Public Health, University of California Berkeley, Berkeley, CA, USA. 
Received: 16 January 2021 Accepted: 23 August 2021

Published online: 06 September 2021

\section{References}

1. UNAIDS. Global HIV \& AIDS statistics-2020 fact sheet. 2020. https:// www.unaids.org/en/resources/fact-sheet\#: :text=38.0\%20million\%20\% 5B31.6\%20million\%E2\%80\%9344.5,AIDS\%2Drelated\%20illnesses\%20in\% 202019. Accessed 12 Nov 2018.

2. Rotger M, Glass TR, Junier T, et al. Contribution of genetic background, traditional risk factors, and HIV-related factors to coronary artery disease events in HIV-positive persons. Clin Infect Dis. 2013;57(1):112-21.

3. Elion RA, Althoff KN, Zhang J, et al. Recent abacavir use increases risk of type 1 and type 2 myocardial infarctions among adults with HIV. J Acquir Immune Defic Syndr. 2018;78(1):62-72.

4. Marcus JL, Neugebauer RS, Leyden WA, et al. Use of abacavir and risk of cardiovascular disease among HIV-infected individuals. J Acquir Immune Defic Syndr. 2016;71(4):413-9.

5. Dorjee K, Baxi SM, Reingold AL, Hubbard A. Risk of cardiovascular events from current, recent, and cumulative exposure to abacavir among persons living with HIV who were receiving antiretroviral therapy in the United States: a cohort study. BMC Infect Dis. 2017;17(1):708.

6. Dorjee K, Choden T, Baxi SM, Steinmaus C, Reingold AL. Risk of cardiovascular disease associated with exposure to abacavir among individuals with HIV: a systematic review and meta-analyses of results from seventeen epidemiologic studies. Int J Antimicrob Agents. 2018;52(5):541-53.

7. Sabin CA, Reiss P, Ryom L, et al. Is there continued evidence for an association between abacavir usage and myocardial infarction risk in individuals with HIV? A cohort collaboration. BMC Med. 2016;14:61.

8. Sabin CA, Worm SW, Weber R, et al. Use of nucleoside reverse transcriptase inhibitors and risk of myocardial infarction in HIV-infected patients enrolled in the D:A:D study: a multi-cohort collaboration. Lancet. 2008;371(9622):1417-26.

9. Desai M, Joyce $V$, Bendavid $\mathrm{E}$, et al. Risk of cardiovascular events associated with current exposure to HIV antiretroviral therapies in a US veteran population. Clin Infect Dis. 2015;61(3):445-52.

10. Opsomer M, Dimitrova D, Verspeelt J, et al. Evaluation of cardiovascular disease risk in HIV-1-infected patients treated with darunavir. Drugs R D. 2018;18(3):199-210.

11. Ryom L, Lundgren JD, El-Sadr W, et al. Cardiovascular disease and use of contemporary protease inhibitors: the D:A: D international prospective multicohort study. Lancet HIV. 2018;5(6):e291-300.

12. Hsue PY, Waters DD. HIV infection and coronary heart disease: mechanisms and management. Nat Rev Cardiol. 2019;16(12):745-59.

13. Bedimo RJ, Westfall AO, Drechsler H, Vidiella G, Tebas P. Abacavir use and risk of acute myocardial infarction and cerebrovascular events in the highly active antiretroviral therapy era. Clin Infect Dis. 2011;53(1):84-91.

14. Brouwer ES, Napravnik S, Eron JJ Jr, et al. Effects of combination antiretroviral therapies on the risk of myocardial infarction among HIV patients. Epidemiology. 2014;25(3):406-17.

15. Young J, Xiao Y, Moodie EEM, et al. Effect of cumulating exposure to abacavir on the risk of cardiovascular disease events in patients from the Swiss HIV cohort study. J Acquir Immune Defic Syndr. 2015;69(4):413-21.

16. IQVIA. IMS health pharmetrics plus data dictionary. 2013. https://tri.uams. edu/wp-content/uploads/sites/80/2015/08/Pharmetrics-Plus-Data-Dicti onary-Jan-2013.pdf. Accessed 19 Jul 2020.

17. Centers for Disease Control and Prevention. HIV-Positive !dults in are in the United States, Medical Monitoring Project, 2009-2014. 2014. https:// www.cdc.gov/hiv/pdf/statistics/systems/mmp/cdc-hiv-mmp-nationaldata-2009-2014.pdf. Accessed 19 Jul 2020

18. Robins JM, Hernan MA, Brumback B. Marginal structural models and causal inference in epidemiology. Epidemiology. 2000;11(5):550-60.

19. Cole SR, Hernan MA. Constructing inverse probability weights for marginal structural models. Am J Epidemiol. 2008;168(6):656-64.

20. Fewell Z, Hernan MA, Wolfe F, Tilling K, Choi H, Sterne JAC. Controlling for time-dependent confounding using marginal structural models. Stand Genomic Sci. 2004:4(4):402-20.

21. Benjamin EJ, Virani SS, Callaway CW, et al. Heart disease and stroke statistics-2018 update: a report from the American Heart Association. Circulation. 2018;137(12):e67-492.

22. Rosenblatt L, Farr AM, Johnston SS, Nkhoma ET. Risk of Cardiovascular events among patients initiating efavirenz-containing versus efavirenzfree antiretroviral regimens. Open Forum Infect Dis. 2016;3(2):ofw061.

23. Eron JJ, Young B, Cooper DA, et al. Switch to a raltegravir-based regimen versus continuation of a lopinavir-ritonavir-based regimen in stable HIV-infected patients with suppressed viraemia (SWITCHMRK 1 and 2): two multicentre, double-blind, randomised controlled trials. Lancet. 2010:375(9712):396-407.

24. Lennox JL, Dejesus E, Berger DS, et al. Raltegravir versus Efavirenz regimens in treatment-naive HIV-1-infected patients: 96-week efficacy, durability, subgroup, safety, and metabolic analyses. J Acquir Immune Defic Syndr. 2010;55(1):39-48.

25. Young L, Wohl DA, Hyslop WB, Lee YZ, Napravnik S, Wilkin A. Effects of raltegravir combined with tenofovir/emtricitabine on body shape, bone density, and lipids in African-Americans initiating HIV therapy. HIV Clin Trials. 2015;16(5):163-9.

26. Santos JR, Saumoy M, Curran A, et al. The lipid-lowering effect of tenofovir/emtricitabine: a randomized, crossover, double-blind, placebocontrolled trial. Clin Infect Dis. 2015:61(3):403-8.

27. Delaney JA, Scherzer R, Biggs ML, et al. Associations of antiretroviral drug use and HIV-specific risk factors with carotid intima-media thickness. AIDS. 2010;24(14):2201-9.

28. Feinstein MJ, Hsue PY, Benjamin LA, et al. Characteristics, prevention, and management of cardiovascular disease in people living with HIV: a scientific statement from the American Heart Association. Circulation. 2019;140(2):e98-124.

29. Elzi L, Erb S, Furrer H, et al. Adverse events of raltegravir and dolutegravir. AIDS. 2017:31(13):1853-8.

30. U.S. Department of Health and Human Services. Guidelines for the use of antiretroviral agents in adults and adolescents living with HIV. October 25, 2018 2018. https://aidsinfo.nih.gov/guidelines/html/1/adult-andadolescent-arv/11/what-to-start--initial-combination-regimens-for-theantiretroviral-naive-patient. Accessed 12 Nov 2018.

31. Kiyota Y, Schneeweiss S, Glynn RJ, Cannuscio CC, Avorn J, Solomon $\mathrm{DH}$. Accuracy of Medicare claims-based diagnosis of acute myocardial infarction: estimating positive predictive value on the basis of review of hospital records. Am Heart J. 2004;148(1):99-104.

32. Hernan MA, Brumback B, Robins JM. Marginal structural models to estimate the causal effect of zidovudine on the survival of HIV-positive men. Epidemiology. 2000;11(5):561-70.

\section{Publisher's Note}

Springer Nature remains neutral with regard to jurisdictional claims in published maps and institutional affiliations. 